\title{
Evanescent Wave Optical Trapping and Sensing on Polymer Optical Fibers for Ultra-Trace Detection of Glucose
}

\author{
Tahereh Azargoshasb, H. Ali Navid,* Roghaieh Parvizi,* and Hadi Heidari
}

Cite This: ACS Omega 2020, 5, 22046-22056

Read Online

ABSTRACT: Graphene sensitization of glucose-imprinted polymer (G-IP)coated optical fiber has been introduced as a new biosensor for evanescent wave trapping on the polymer optical fiber to detect low-level glucose. The developed sensor operates based on the evanescent wave modulation principle. Full characterization via atomic force microscopy (AFM), Fourier transform infrared (FTIR) spectroscopy, X-ray diffraction (XRD), scanning electron microscopy (SEM), Raman spectroscopy, high-resolution transmission electron microscopy (HRTEM), and $\mathrm{N}_{2}$ adsorption/desorption of as-prepared G-IP-coated optical fibers was experimentally tested. Accordingly, related operational parameters such as roughness and diameter were optimized. Incorporating graphene into the G-IP not only steadily promotes the electron transport between the fiber surface and as-proposed G-IP but also significantly enhances the sensitivity by acting as a carrier for immobilizing G-IP with specific imprinted cavities. The

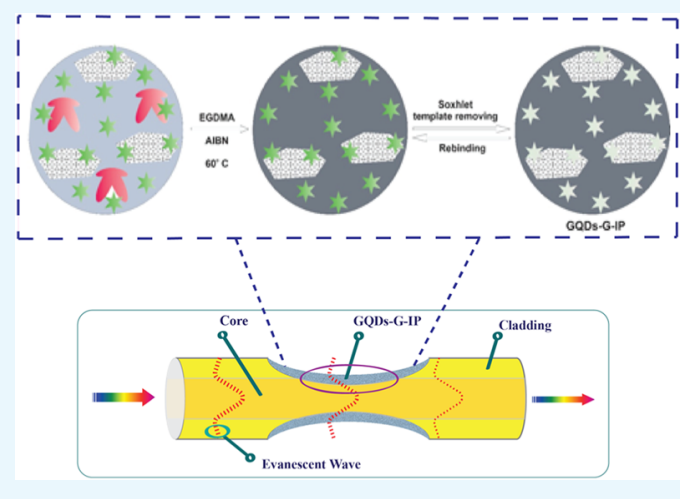
sensor demonstrates a fast response time $(5 \mathrm{~s})$ and high sensitivity, selectivity, and stability, which cause a wide linear range (10-100 $\mathrm{nM})$ and a low limit of detection $(\mathrm{LOD}=2.54 \mathrm{nM})$. Experimental results indicate that the developed sensor facilitates online monitoring and remote sensing of glucose in biological liquids and food samples.

\section{INTRODUCTION}

Glucose quantification is of great interest in medical, biological, and food industries. ${ }^{1-4}$ In the context of healthcare and life quality, consumption of food rich in carbohydrates becomes a growing concern by gaining the status of diabetes development in patients. Hence, to maintain and impede the development of diabetes, people need to consume products of low calories or non-nutritive sweeteners and sugar-free foods ( $0.5 \mathrm{~g}$ sugar per serving). ${ }^{5}$ Therefore, low-level glucose detection based on an inexpensive and sensitive sensor is of a huge commercial healthcare significance and favor with a potential of direct application in diverse matrixes. Detection of micro- and millimolar glucose concentrations has been widely reported, while a few types of sensors such as radio frequency resonatorbased integrated passive device, ${ }^{6}$ photometer based on metal waveguide capillary, ${ }^{7}$ and photoelectrical detection using $\mathrm{TiO}_{2}$ nanowires with surface-functionalized glucose oxidase ${ }^{2}$ have been developed as an effort to detect glucose at very low levels. ${ }^{1,3,4,8,9}$ It is still highly desired to develop a new probe to improve sensitivity, selectivity, and feasibility and to refine a tedious process for active material preparation. Due to the excellent features of sensitivity, selectivity, low cost, and miniaturized sensing probes, electrochemical signal transduction-coupled $^{10,11}$ optical fiber sensors have been the center of attention in the fields of physics, optics, and analytical chemistry. ${ }^{12}$ Such innovative technology and mechanism have been emerged to monitor and detect glucose in real time, including sensors based on electrochemical signal trans- duction $^{10,11}$ and optical fiber sensors. ${ }^{12}$ Optical fibers have been utilized in different categories of biosensors including fluorescence, refraction, and absorption with the operating principle of evanescent wave absorbance (EWA). ${ }^{12-15}$ In an optical fiber transducer-based sensor, within the unclad region, light leaks out from the core to the less dense medium (e.g., solutions), thus generating evanescent wave (EW) at the interface of the core and the surrounding environment. The amplitude of EW decreases exponentially from the core into the aqueous solution sample, which represents the changes corresponding to the analyte molecules adsorbed, adhered, or bound to the chemically modified optical fiber surface. Therefore, it is clear that improving the strength of EW within the unclad region essentially affects achieving high sensitivities and low detection limits; thus, research on the synergy between absorbance or attenuation nanostructure layers and optical fiber intrinsic properties has gained momentum. In the past decade, enzyme-linked optical fiber was established to develop a fast, sensitive, selective, and reliable detection device for glucose concentration, while these biosensors suffer from

Received: April 25, 2020

Accepted: August 7, 2020

Published: August 25, 2020 
Scheme 1. Schematic Diagrams of the GQDs-G-IP Synthesis Procedure ${ }^{1}$
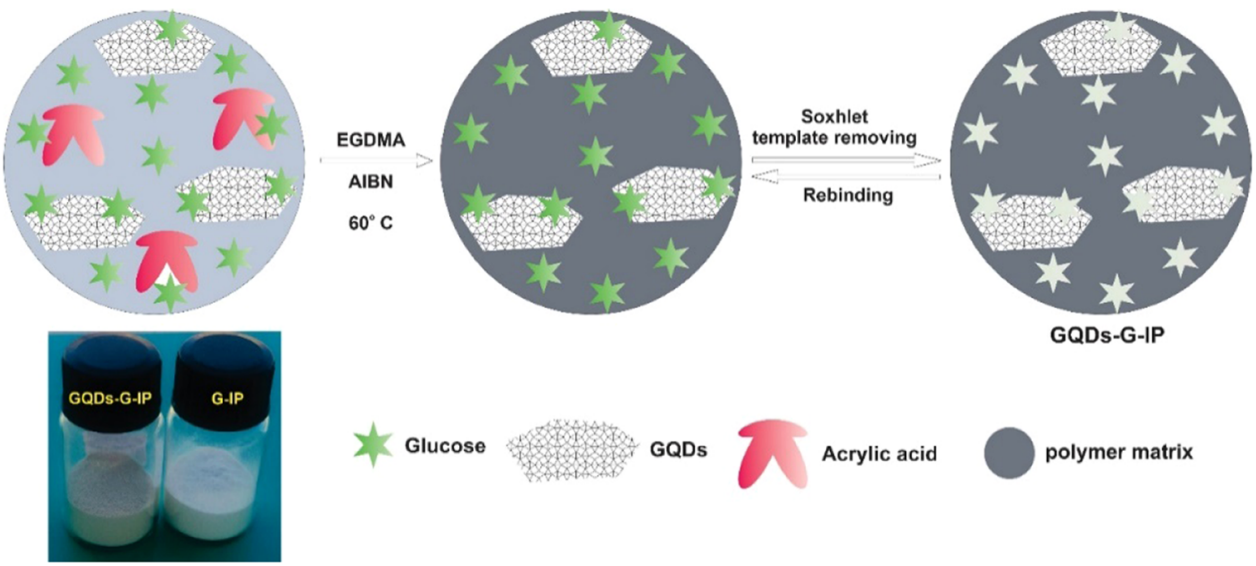

Glucose
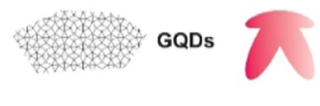

Acrylic acid

polymer matrix

${ }^{1}$ Inset shows photographs of GQDs-G-IP and G-IP

instability, high cost, and complicated measurement procedures involving enzyme immobilization and purification steps. ${ }^{16}$ To overcome these limitations, a nonenzymatic and selective technique of molecularly imprinted polymers (MIPs) has been exploited as an artificial and tailor-made receptor with high affinity and selectivity toward a specified target molecule. ${ }^{17-19}$ This useful specification stems from the presence of selective cavities with a three-dimensional (3D) structure that matches in complementary shape, size, and chemical functionality to target known as "imprinted" binding sites. $^{20,21}$ However, MIP functionality suffers from low optical activity, medium surface area of MIPs, and their aggregation and collapse stemming mainly from intramolecular interaction. To overcome these limitations, the MIP growth on other surfaces such as semiconductors, ${ }^{22}$ perovskites, ${ }^{23}$ and conducting polymers ${ }^{22,24}$ or their sensitization via organic and/or inorganic quantum dots, noble-metal nanoparticles, and graphene or graphene oxide as a carbon-based source is of great interest. $^{25-29}$ Supporting the properties of both MIPbased materials by the aforementioned materials for providing a larger surface area for the diffusion of reactants onto the active sites enhances the adsorption performance. In graphene quantum dots (GQDs) with two-dimensional (2D) layered structures, unique features of zero rest mass of its charged carriers, high solubility and dispersibility, as well as higher carrier mobility have been exploited to improve the optical activity and surface of polymeric-based MIPs. ${ }^{25-27}$ Incorporation of GQDs enlarges electron transfers and surface area of MIPs, leading to an intensified sensitivity as well as introducing a nonenzymatic selective sensing layer. ${ }^{30}$ Moreover, graphene in dot size can be impregnated into the polymeric network of the proposed MIP during the polymerization process. In this case, acrylic acid (AA) monomer can be interacted and grown on $\mathrm{COOH}$ and $\mathrm{OH}$ groups of GQD by covalent and $\mathrm{H}$ bonds.

The latest work on enzyme-based optical fiber sensors coated with a carbon quantum dot-glucose oxidase/cellulose acetate complex sensitive film has been reported in the nanomolar range of $10-100 \mathrm{nM}^{31}$ Therefore, in the present study, a simple and rapid optical sensing method was developed to prepare a GQD-sensitized molecularly imprinted polymer and subsequently coated on evanescent wave optical fiber as a new biosensor for trace-level detection of glucose as a target molecule. The used GQD was coupled with nanosheets, which were synthesized from natural coal through some simple treatments of chemical oxidation and centrifugation, reported in our previous work. ${ }^{32}$ Operational parameters, fiber diameters, different surface roughness, and sensing performance experiments were compared to find the optimized condition.

\section{EXPERIMENTAL METHODS}

2.1. Materials and Apparatuses. Ethylene glycol dimethacrylate (EGDMA), acrylic acid (AA), dimethyl sulfoxide (DMSO), 2,2'-azo-bis-isobutyronitrile (AIBN), glucose, and $\mathrm{N}$-methy1-2-pyrrolidone (NMP) were purchased from Merck Company (Darmstadt, Germany) and used without any further purification. Fourier transform infrared (FTIR) spectra were recorded in the $400-4000 \mathrm{~cm}^{-1}$ range with a PerkinElmer UATR Two spectrometer according to the $\mathrm{KBr}$ pellet technique by mixing about $1 \mathrm{mg}$ of the sample and $100 \mathrm{mg}$ of $\mathrm{KBr}$. Atomic force microscopy (AFM) image of the optical fiber surface was achieved using an Alpha 300 (Wintec, Germany). The surface morphology of the as-prepared functionalizing powders was studied by a scanning electron microscope (SEM, ZEISS Sigma A = SE2, Germany). Nitrogen adsorption analysis of Brunauer-Emmett-Teller (BET) was carried out using Belsorp mini П, Japan.

2.2. Materials Preparation. The proposed glucoseimprinted polymer (G-IP) in the presence and absence of GQDs was prepared as follows: $170 \mu \mathrm{L}$ of functional monomer (AA) and $0.5 \mathrm{mmol}(0.09 \mathrm{~g})$ of glucose were dissolved in 10 $\mathrm{mL}$ of DMSO and stirred for $1 \mathrm{~h}$. Then, $1.5 \mathrm{~mL}$ of EGDMA and $0.008 \mathrm{~g}$ of AIBN were added to the above dispersion under nitrogen purging for $10 \mathrm{~min}$ to remove dissolved oxygen. Then, the obtained mixture was heated to $60{ }^{\circ} \mathrm{C}$ for $24 \mathrm{~h}$ for AIBN initiator activation and starting polymerization. Finally, the obtained bulk polymers were ground and washed two times by water and methanol to remove any nonpolymerized monomers and DMSO, while template removal was conducted using Soxhlet extraction with methanol/acetic acid (7:3) three times (each time $16 \mathrm{~h}$ ). Additionally, a nonimprinted polymer (NIP) was synthesized using the same procedure without glucose as template molecules. For comparison, G-IP in the absence of GQDs was synthesized similar to the above procedure and 0.8 $\mathrm{g}$ of GQDs was added. All steps for materials preparation are illustrated in Scheme 1.

2.3. Preparation of Sensing Probe and Experimental Evanescent Wave-Based Setup. Different core diameters of 
the multimode plastic-optical fiber (1.0, 0.75, and $0.50 \mathrm{~mm})$ were used, and the protective sleeve of the bare the fibers was removed mechanically via a fiber stripper of a fiber length 1.4 $\mathrm{cm}$ length; then, this segment as the sensing region was unclad mechanically by a homemade machine (Figure $1 \mathrm{~b}$ ). In this

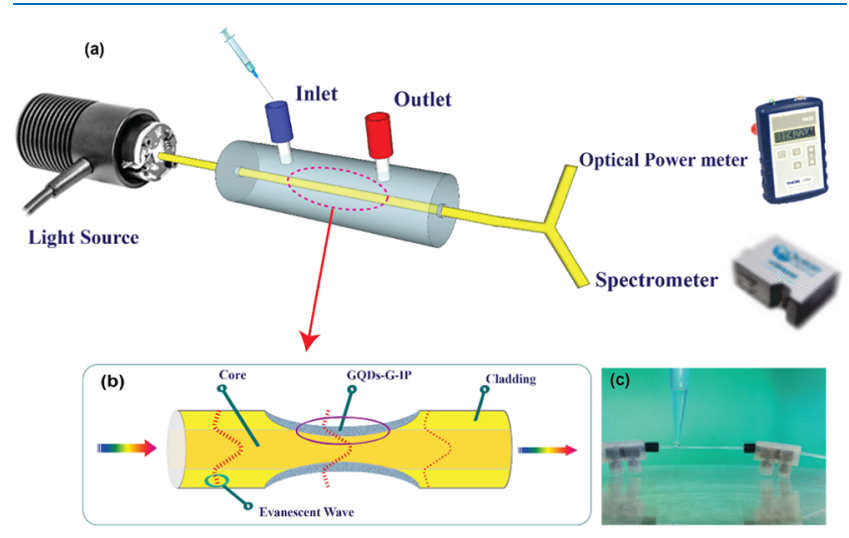

Figure 1. (a) Experimental evanescent wave-based setup with the images of the evanescent wave in the sensing region and drop-casting technique for coating the unclad region; (b) the emerged evanescent wave in the unclad region of optical fiber; and (c) the drop-casting of G-IP on the optical fiber.

step, the final surface of the optical fiber was polished via several sandpapers to reach different roughnesses. The unclad portion of the fiber was rinsed in double-distilled water two times. To coat the sensing segment on optical fiber, a mixture of the synthesized powders was prepared as follows: $0.015 \mathrm{~g}$ of NIP (G-IP, GQDs-G-IP) and $2 \mathrm{~mL}$ of NMP were mixed at room temperature for $12 \mathrm{~h}$. Then, $0.005 \mathrm{~g}$ of poly(vinylidene difluoride) (PVDF) was added to the mixture and dispersed in an ultrasonic bath for $30 \mathrm{~min}$. After that, the as-prepared mixture was drop-cast four times the unclad portion of the fiber and activated at $45{ }^{\circ} \mathrm{C}$ for $10 \mathrm{~min}$ (see Figure 1c). ${ }^{33} \mathrm{~A}$ white-light source lamp (MBB1L3 M00465970, Thorlabs Co.) was used to launch a broad-band light to one terminal end of the fiber in the visible region. The other terminal end of the fiber was connected to a fiber-optic spectrometer (HR4000CG-UV-NIR from Ocean Optics, Florida) or an optical power meter (Thorlabs Co.). A transparent capillary tube was used as a cell, in which fiber was fixed with ultraviolet (UV) glue in the two ends of the tube. Thereby, the experimental arrangement used for glucose sensing is illustrated in Figure 1a, showing the two ends of the sensing probe attached to the light source and detector to record absorbance and attenuation of the evanescent wave with the help of a computer interfaced with the spectrometer. The unclad segment of the fiber was fixed in a cell enabled to deliver and remove background water and also glucose solution. Each measurement of $1.2 \mathrm{~mL}$ of analyte solution by a very soft syringe was poured into the cell, and real-time intensity changes were recorded.

\section{RESULTS AND DISCUSSION}

3.1. Material Characterizations. In our previous work, we managed to prepare biocompatible nitrogen-doped GQDs using simple treatments of chemical oxidation and centrifugation separation method. ${ }^{32}$ The versatility of the prepared samples as sensitizing agents for glucose-imprinted polymer was developed, and polymer optical fiber was coated as well as its ability to detect low-level concentration of glucose was established. The transparency observed in the HRTEM images (Figure 2a,b) indicates the few-layered structure and good dispersion of two-dimensional graphene layers or sheets. Some other segments show obvious quantum-dot-coupled and wrinkled structures with some compact folds that denote aggregations of graphene layers. In the Raman spectrum (Figure 2c), obvious bands are located around 1590 and 1383 $\mathrm{cm}^{-1}$, which were generally assigned as the D band and G band, representing the structural defects and vibration of $\mathrm{sp}^{2}$ hybridized graphitic domains, respectively.

3.1.1. Surface Morphology. The surface morphologies of NIP, G-IP, and GQDs-G-IP were analyzed by field emission scanning electron microscopy (FESEM) (Figure 3), which shows a uniform semispherical polymeric porous structure with rough surfaces and agglomerated portion. Compared to the morphology of the G-IP particles, the GQDs-G-IP showed more pores and roughness, while NIP exhibited a different morphology with a smooth spherical shape. The formation of cavities for GQDs-G-IP implying the successful combination of G-IP- and GQD-coupled nanosheets can lead to a high surface area and an increase in the functionality of the optical property. GQD due to its dispersibility and solubility was well impregnated in the MIP structure and inhabited from the collapse and agglomeration of MIP. However, the intermolecular interaction in the absence of GQD causes the aggregation of MIP layers.

3.1.2. FTIR Analysis. FTIR spectroscopy was carried out to specify the functional groups in the as-prepared samples before and after the glucose extraction step (see Figure 4). As shown, in the GQDs FTIR spectrum, the peak at $1628 \mathrm{~cm}^{-1}$ was attributed to the stretching vibration of $\mathrm{C}=\mathrm{O}$ groups. Also, the absorption corresponded to symmetric stretching vibration at $\sim 1445 \mathrm{~cm}^{-1}$ and COO- antisymmetric stretching vibration
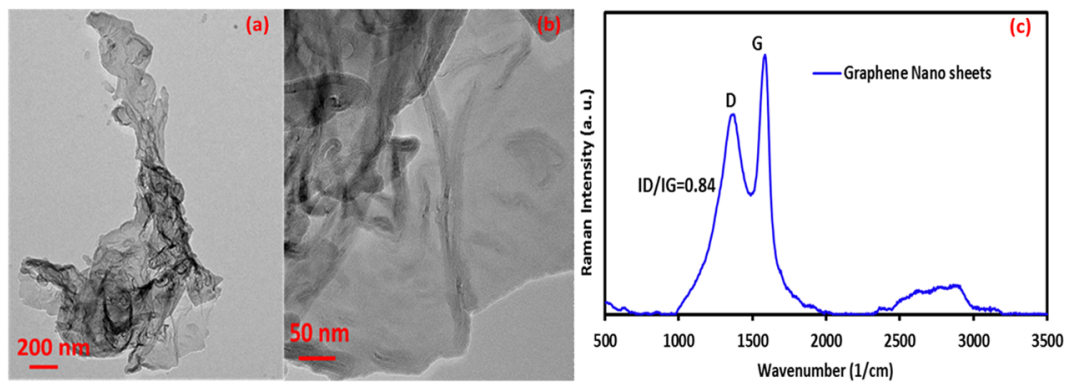

Figure 2. HRTEM images in different magnifications ( $a, b)$ and Raman spectra of the as-synthesized of nitrogen-doped GQD-coupled nanosheets (c), taken from ref 32 . 


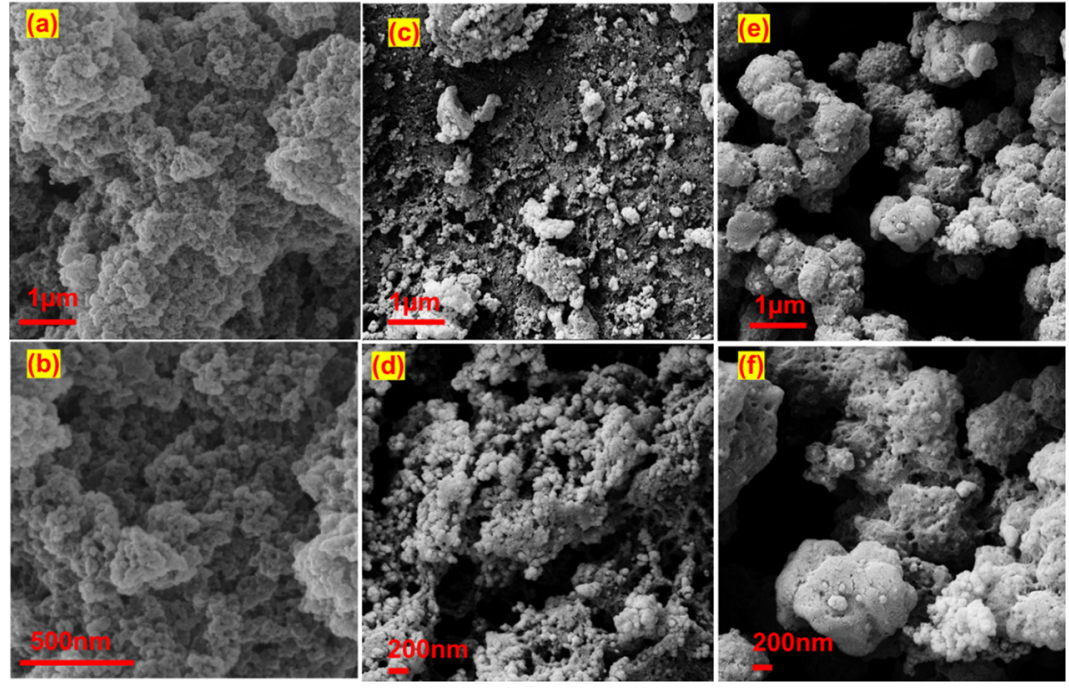

Figure 3. FESEM images of NIP (a, b), G-IP (c, d), and GQDs-G-IP (e, f) in two magnifications.

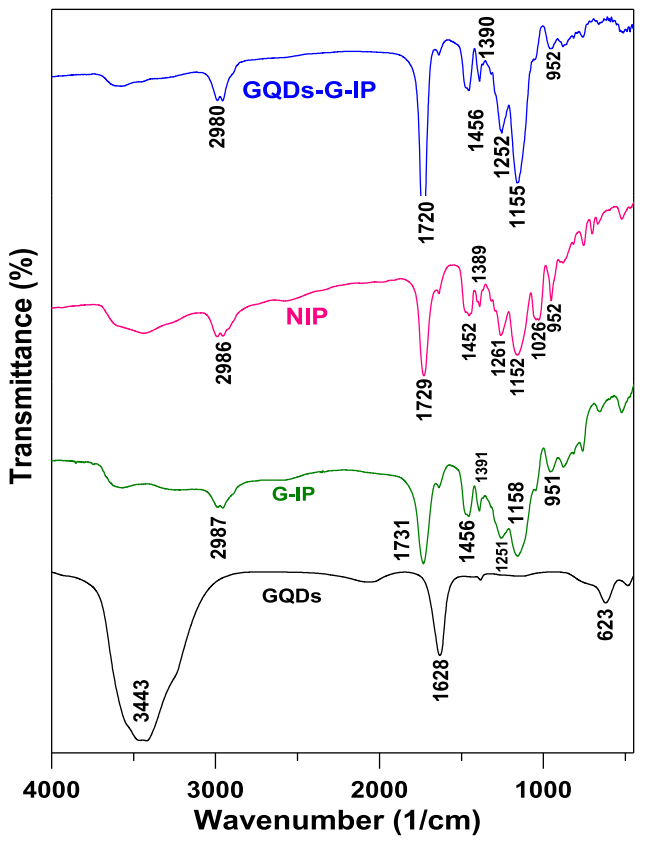

Figure 4. FTIR spectra of the as-prepared samples. at $3443 \mathrm{~cm}^{-1}$. In G-IP and NIP, a wide vibration absorbance occurs in the region of $1700-1750 \mathrm{~cm}^{-1}$, which is related to the stretching mode of the $\mathrm{C}=\mathrm{O}$ groups in EGDMA and $\mathrm{AA}$, while in GQDs-G-IP, due to the chemical interaction between GQDs and G-IP, the corresponding $\mathrm{C}=\mathrm{O}$ peak shifted to a lower wavenumber. In addition, the $\mathrm{C}-\mathrm{H}$ stretching peak was also observed at around $2932 \mathrm{~cm}^{-1}$, which can be attributed to the presence of the methylene group in both AA and EGDMA of G-IP, NIP, and GQDs-G-IP. The related glucose peaks in GQDs-G-IP and G-IP were of low intensity at NIP, and even some of the peaks were destroyed, which confirmed the successful preparation of the proposed samples.

3.1.3. BET Analysis. Brunauer-Emmett-Teller (BET) surface area and Barrett-Joyner-Halenda (BJH) pore size and volume analysis were further investigated (Figure 5), which confirm the formation of $\mathrm{H} 2$ pore structure and type IV isotherm for all as-prepared materials. The specific surface areas of $98.478,118.87$, and $130.23 \mathrm{~m}^{2} / \mathrm{g}$ were obtained for NIP, G-IP, and GQDs-G-IP, respectively. The pore size of the samples with a mesoporous structure was obtained in the range of $2-5 \mathrm{~nm} .{ }^{34}$ Thus, it is obvious that GQDs-G-IP showed the largest BET surface area, pore volume, and pore size due to the existence of graphene nanosheets and its effect on the polymer growth and homogenization of its structure and density surface of a porous structure. ${ }^{10}$ Generally, a higher total pore volume of GQDs-G-IP causes a higher sample loading capacity and
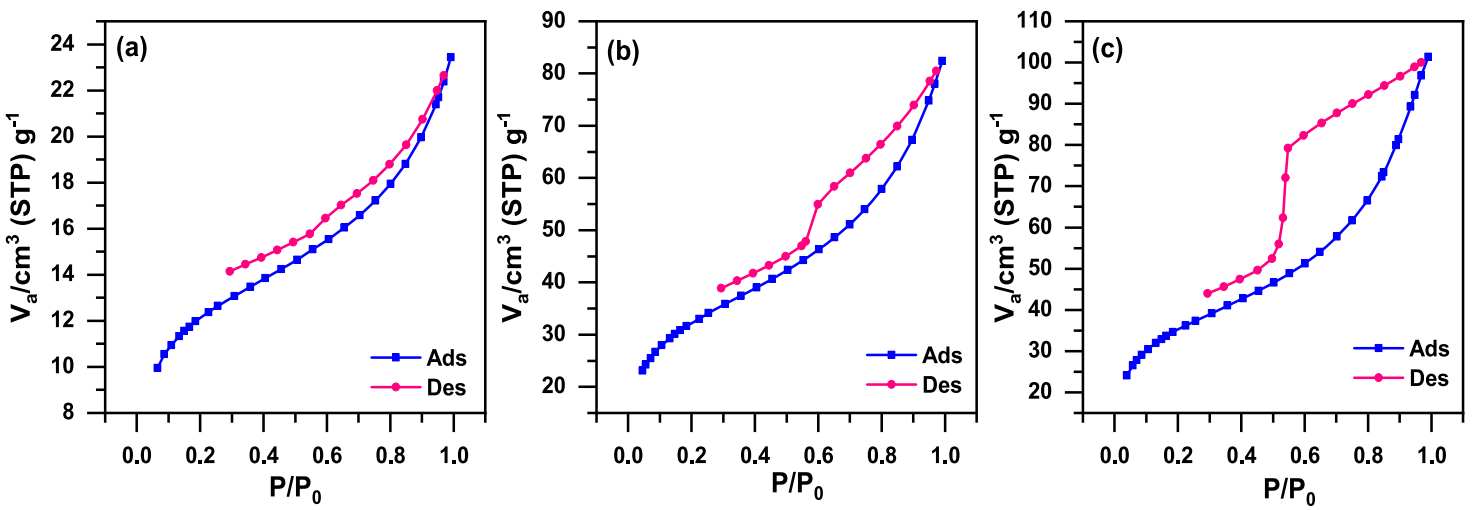

Figure 5. $\mathrm{N}_{2}$ adsorption-desorption for (a) NIP, (b) G-IP, and (c) GQDs-G-IP samples. 
promotes mass transfer between the GQDs-G-IP particles and analyte solution.

3.2. Spectroscopic Investigation of the Sensing

Probe. To evaluate the practical applicability of this sensing probe, effects of operational parameters including spectral transmission, surface roughness of the etched optical fiber, and temperature were investigated on sensor performance. Three main procedures were considered and done during each test; first, to carry out reliable glucose tests by eliminating the impact of background water on the sensitivity, in each measurement, the glucose solution was added to the optical fiber immersed in the background DI water, so the light variation encountered under this act was recorded according to the underlying concept of sensitivity. Moreover, only one sensing probe was experimentally studied under the whole glucose solution ranging from 10 to $2000 \mathrm{nM}$ by successive measurement to check the sensing performance. Third, in the present work, after each measurement, DI water was poured into the flow cell, and after $10 \mathrm{~min}$ waiting time, the adsorbed glucose was removed and washed from the functionalized coating layer.

The proposed sensor operates based on the intensity modulation, which modifies the evanescent field scattered within the sensing region. As the refractive index (RI) of the surrounding environment increases (by immersing the fiber from air in distilled water), the coating layer was reacted with water molecules. Consequently, the RI of the active cladding changes due to the physically and chemically adsorbed water molecules on the surface of the coating layer. Since the evanescent wave within active clad is responsible for a reaction between the sensing probe and the surrounding environment, it would be manipulated according to the RI changes, and so, the alteration in the evanescent wave affects the output light intensity. The transmitted optical power spectra of the bare NIP-, G-IP-, and GQDs-G-IP-coated optical fiber as sensing probes were immersed in air and background solution DI water, and the spectral responses were compared over the visible light range. The bare fiber indicated a reverse behavior of increasing intensity in comparison to the functionalized sensing probes representing decreasing intensity, as shown in Figures 6 and 7, under dry and wet environment conditions. The dynamic responses of NIP-, GIP-, and GQDs-G-IPcoated optical fibers are illustrated in Figure 7, with a similar behavior of shifting down of spectrum indicating an appreciable loss in transmitted light intensity. Indeed, in the case of bare optical fiber, a water environment with RI of 1.3 (more than that of air) as a closer RI to the core of fiber acts as a cladding and thereby permits stronger light guides through the fiber, so the output light intensity increased. In other words, once the bare fiber was dipped in water, in the sensing region, the RI difference between the core and the ambient environment decreases, leading to less leakage and attenuation of light. However, for the functionalized optical fiber probes, the evanescent wave increases due to the more leakage and scattering of light within the sensing region in the wet active clad. From the spectra of sensor response (inset of Figure 7), it can be seen that the highest intensity variation emerged at the wavelength of $578 \mathrm{~nm}$ with the maximum intensity and so the following sensor measurements were performed at this particular wavelength.

In the current study, the capability of high RI coating layers to induce RI contrast in the sensing region was investigated numerically. We have conducted a $2 \mathrm{D}$ analysis of the mode

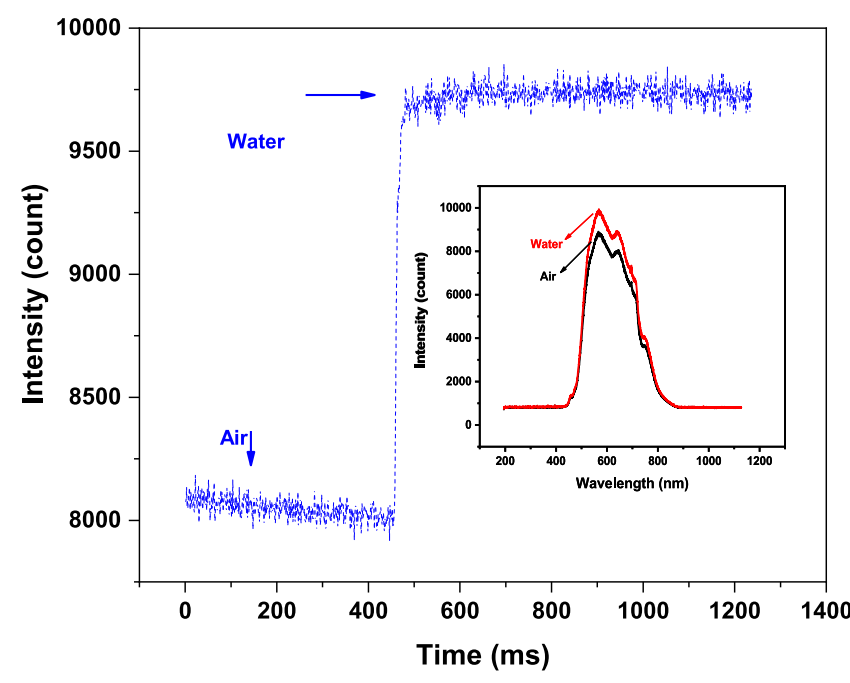

Figure 6. Time response curves of bare optical fiber ( $1 \mathrm{~mm}$ diameter) (the inset shows the transmitted light spectrum once it immersed in water).

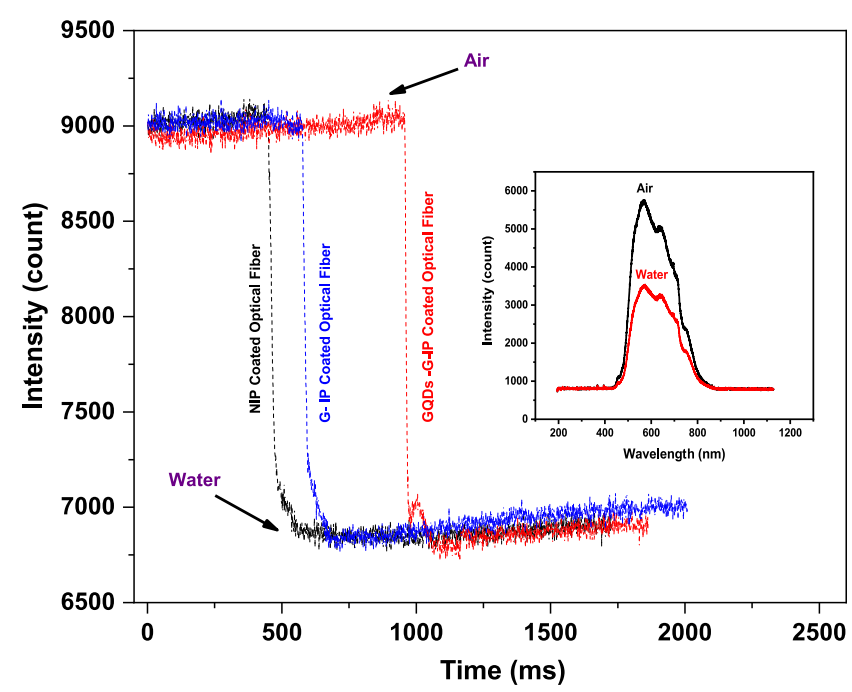

Figure 7. Time response curves of NIP-, G-IP-, and GQDs-G-IPcoated optical fibers (the inset shows the transmitted light spectrum of the G-IP-coated optical fiber under dry and wet conditions).

structure and the electromagnetic field modes along the transverse plane of an unclad polymer fiber using the mode analysis utilities of finite-element analysis-based software. The simulation wavelength was set to $570 \mathrm{~nm}$ (as used in Section $2)$; the square blue area represents the glucose sensing environment including optical fiber and surrounding glucose solution, and the outer edge of the fiber is separated by a layer of G-IM or GQDs-G-IP. The optical parameter RI of polymer optical fiber (POF) (poly(methyl methacrylate)), G-IP (based on acrylic acid polymers), GQDs-G-IP, and glucose solution was set to $1.49,1.508,2.3+1.27 \mathrm{i}$ and $1.34,{ }^{35}$ respectively, to numerically simulate effective indices modal and intensity patterns. As the thickness of G-IP and GQDs-G-IP is very thin, about less than $10 \mu \mathrm{m}$ (Figure $\mathrm{S} 1$, relative to the optical fiber diameter), and more emphasis was given to the RI parameter to trap the evanescent field, it is reasonable to neglect the effect of the sensing coated layers in the analysis and to focus on the mode distribution of the modes within the core and sensing region. The electric field norm distribution of 

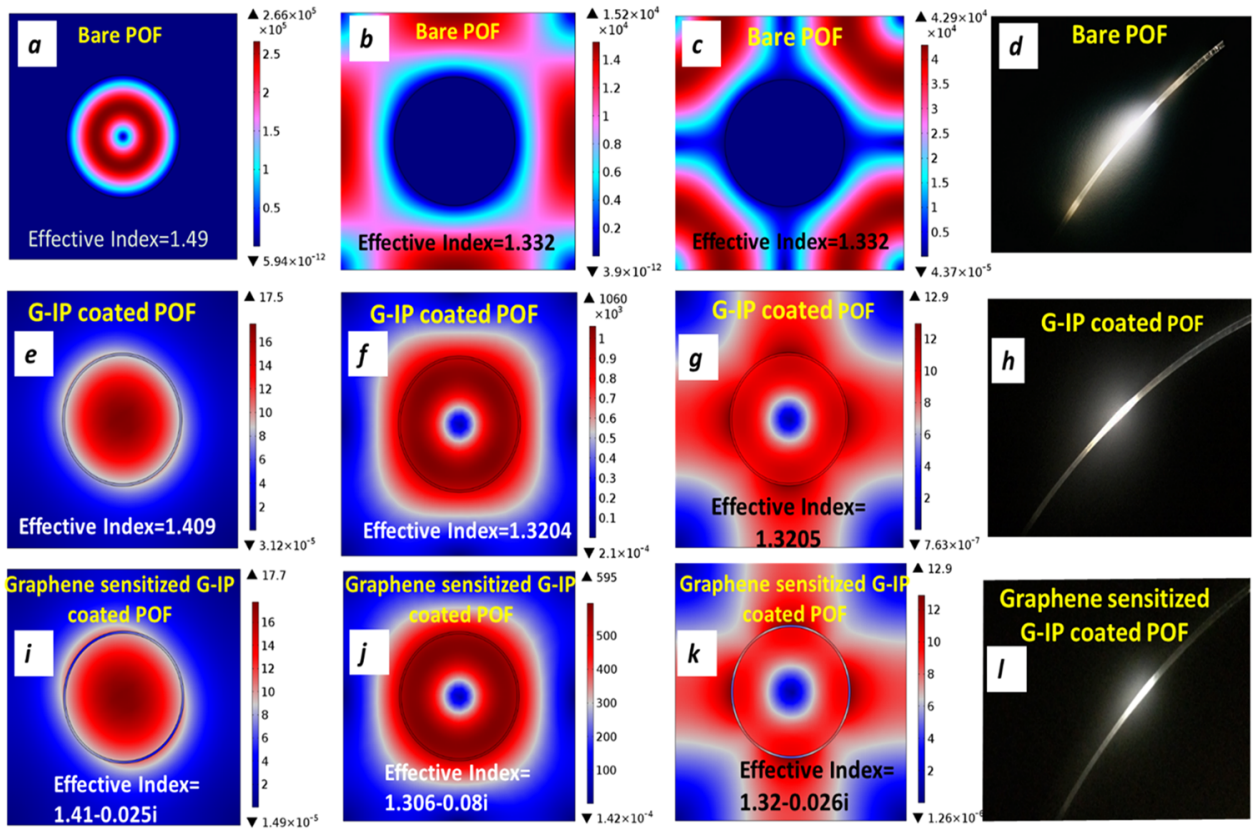

Figure 8. Surface diagram of the electric field norm distribution of the modes of the clad-removed bare fiber (a-d), G-IP-coated fiber (e-h), and the proposed GQDs-G-IP-coated fiber (i-l).
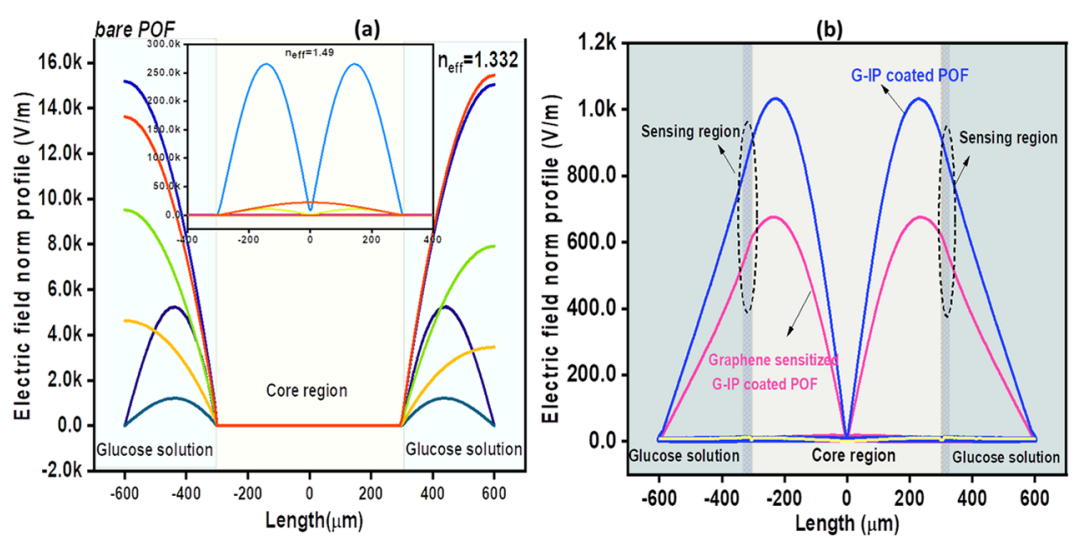

Figure 9. Line graph of evanescent wave leakages from the core into the surrounding environment interface from the bare (a) and coated optical fiber (b).

the fundamental modes within the proposed layer-coated fiber is demonstrated for the cross-sectional view of the bare and sensing layer-coated fibers for various effective RIs in Figures 8 and 9. It can be inferred from the plot that the effective IR for the bare optical fiber is appreciably distinct as 1.49 and 1.332 related to the core and surrounding environment, respectively. This numerical analysis shows that the fundamental modes of bare optical fiber can be well bound in the core region with the exact RI of 1.49 , while the outer region pattern of modes is quite separated from the interface surface, as shown in Figure $8 \mathrm{a}-\mathrm{c}$. The impact of the RI of the coated layer is notable, which can be observed clearly in Figure 8d,h,I. The observed light in the unclad region of fiber would reduce from bare to the high RI layer-coated fibers.

Moreover, it is clear from comparing the figures that the energy of the cladding mode is more concentrated in a position near the core in the case of the G-IP and GQDs-G-IP-coated fibers (Figure 8f,j) than in the case of the bare fiber (Figures $8 \mathrm{~b}$ and 9a). The G-IP cladding on the outside of the unclad fiber forms a high RI ring so that the energy of the high-order cladding mode is concentrated in the vicinity of the coated layer, which is acting as the sensing layer, as shown in Figures $9 \mathrm{~b}$ and S2, giving rise to the stronger evanescent wave and higher detecting features of the sensor. This layer supports not only optical modes confined in the boundary between the core and the coated layer but also some optical modes guided by the core due to the high index of refraction originating from light trapping introduced as evanescent wave (in the line graphs of Figure 9b).

\section{RESULTS OF SENSING RESPONSE EXPERIMENTS AND DISCUSSION}

The evanescent field strength can depend on some determining factors like input light intensity as well as specifications including diameter size and surface roughness of the optical fiber. So, to effectively calibrate and assess the sensing performance, the normalized response (NR) and sensitivity $(S)$ were, respectively, determined by $\mathrm{NR}=\left(I_{\text {out }}-\right.$ $\left.I_{\text {in }}\right) / I_{\text {in }} \times 100 \%$ and $S=\Delta \mathrm{NR} / \Delta \mathrm{GC}$, where $I_{\text {in }}$ is the launched light intensity into the optical fiber, $I_{\text {out }}$ is the intensity of the 

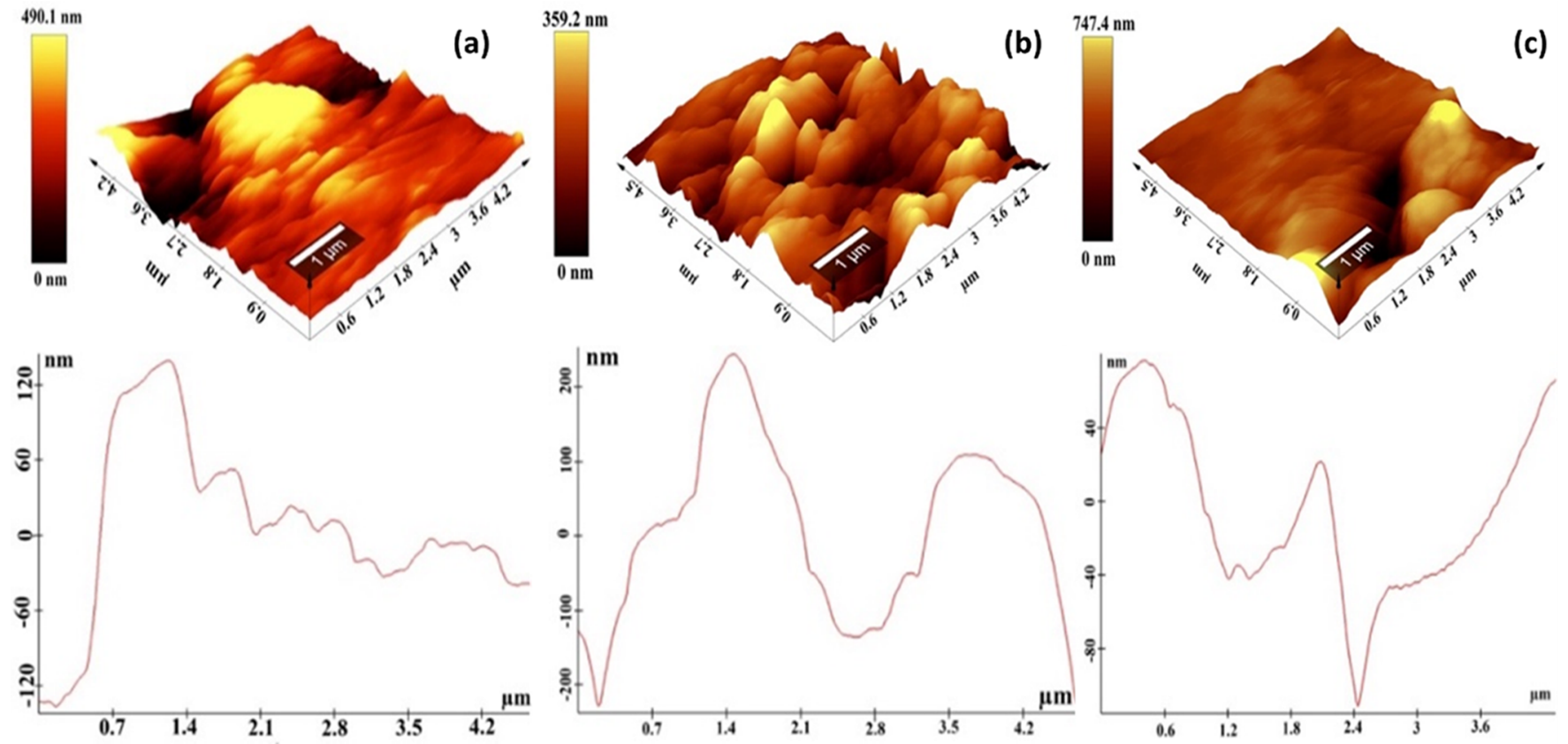

Figure 10. AFM analysis for showing the roughness of optical fiber surfaces polished with (a) 1000, (b) 1500, and (c) 2000 grit sandpaper.
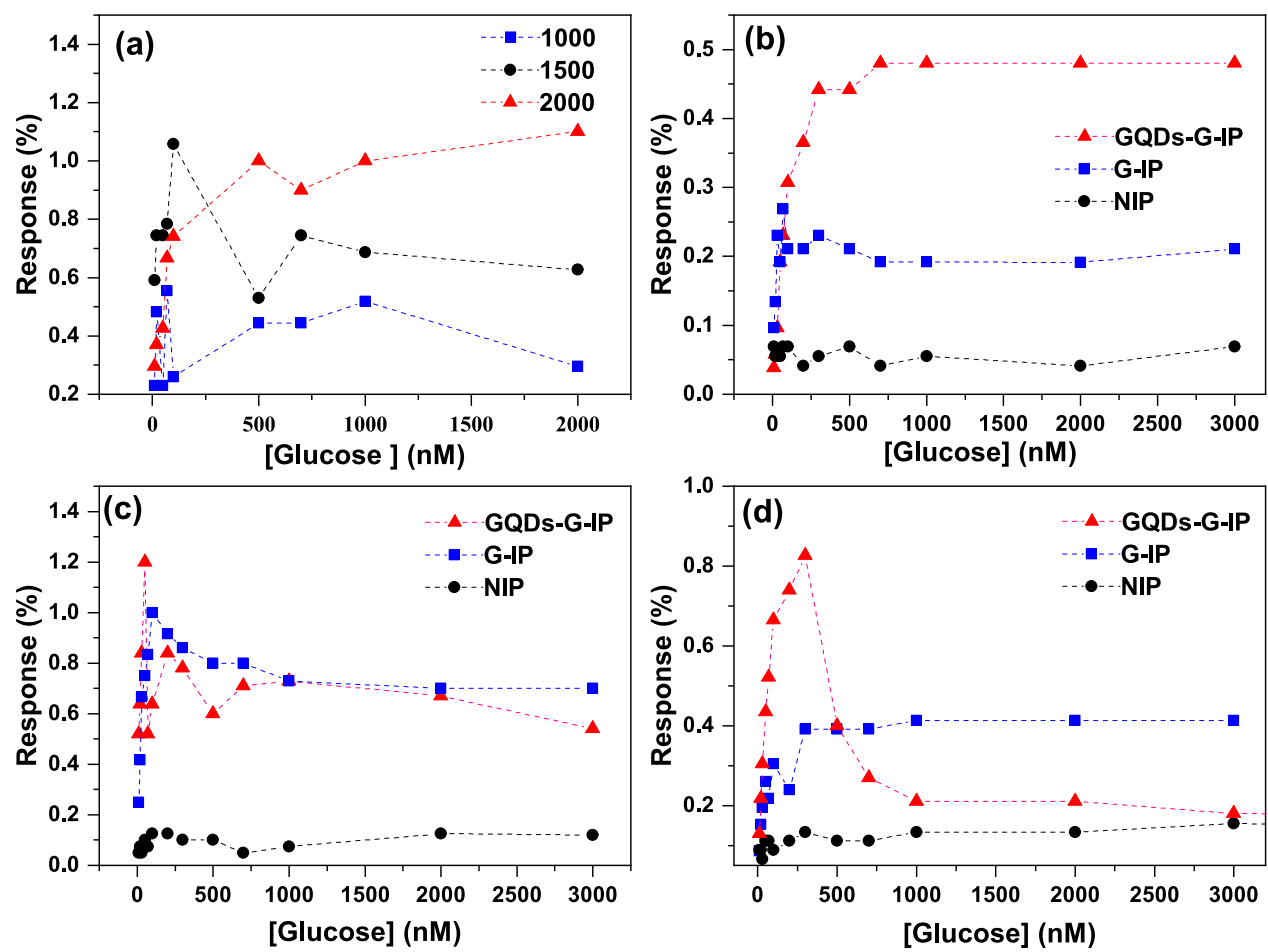

Figure 11. Sensing performance experiments of G-IP in different roughnesses of optical fiber surfaces polished with 1000, 1500, and 2000 grit sandpaper (a) and GQDs-G-IP-POF in different fiber diameters of $0.5 \mathrm{~mm}(\mathrm{~b}), 0.75 \mathrm{~mm}$ (c), and $1.0 \mathrm{~mm}(\mathrm{~d})$.

output light transmitted through optical fiber at every glucose concentration, $\Delta \mathrm{NR}$ is the relative variation in the transmitted light intensity for the sensor, and $\Delta \mathrm{GC}$ is the change of glucose concentration. Although the surface roughness effects in the sensing region on the sensitivity of the evanescent wave-based optical fiber sensor have been investigated, ${ }^{36,37}$ the contribution of this effect with the G-IP coating to the sensitivity has not been checked. Herein, to investigate the effect of optical fiber surface roughness on the sensing performance, three G-
IP-coated optical fibers with different roughness were prepared.

As illustrated in Figure 10, the fibers were surface-scanned over $5 \mu \mathrm{m} \times 5 \mu \mathrm{m}$ for the samples polished with 1000, 1500, and 2000 grit sandpaper. 3D AFM images (see Figure 10) indicate that rougher sandpaper resulted in a surface with higher roughness. For the characterization of the prepared probes fixed in the flow cell, the variation of light intensity as a function of concentrations of glucose solution was recorded. To this aim, glucose solutions with different concentrations 

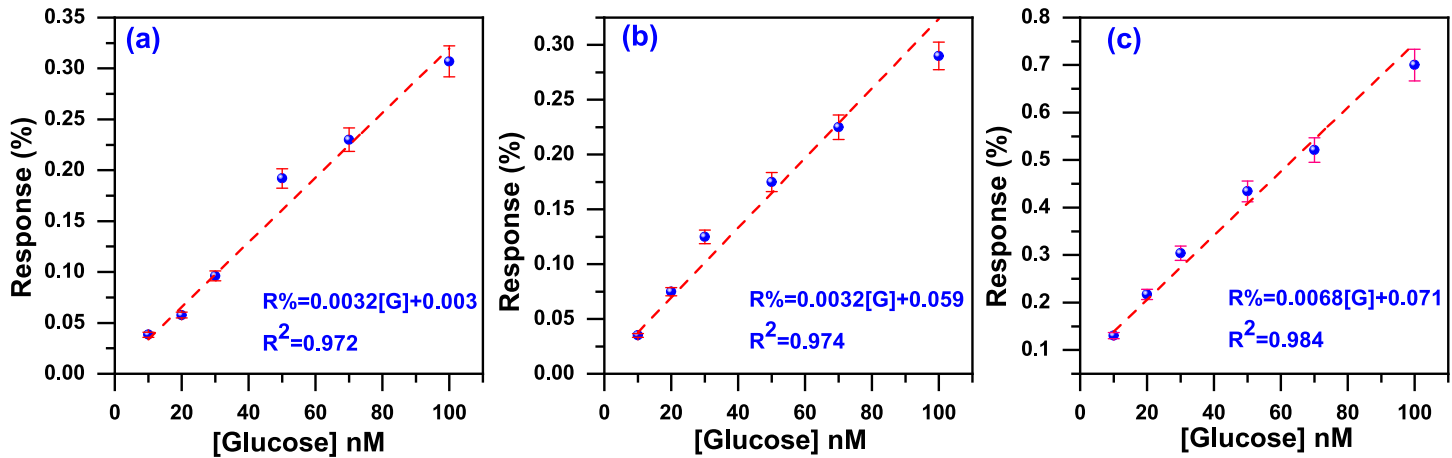

Figure 12. Effect of different optical fiber diameters of $0.5 \mathrm{~mm}(\mathrm{a}), 0.75 \mathrm{~mm}(\mathrm{~b})$, and $1.0 \mathrm{~mm}(\mathrm{c})$ on the sensing performance of probe within the trace-level detection of glucose.

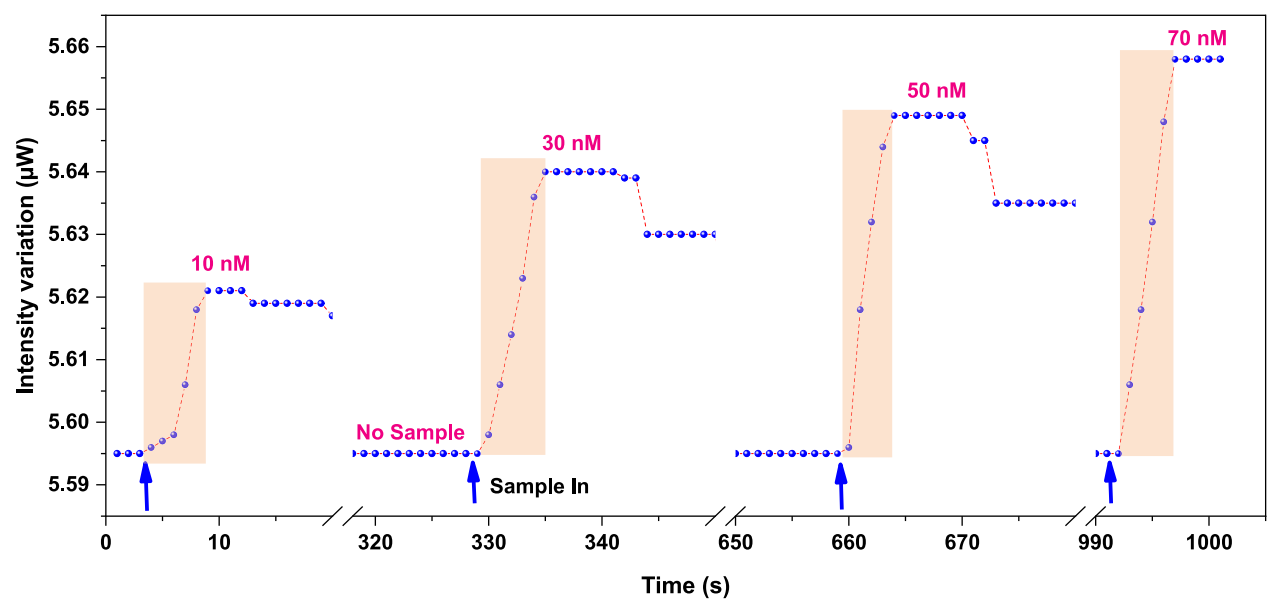

Figure 13. Variation in normalized transmitted power in the case of GQDs-G-IP-coated POF with time during the presence and absence of glucose solution in the flow cell.

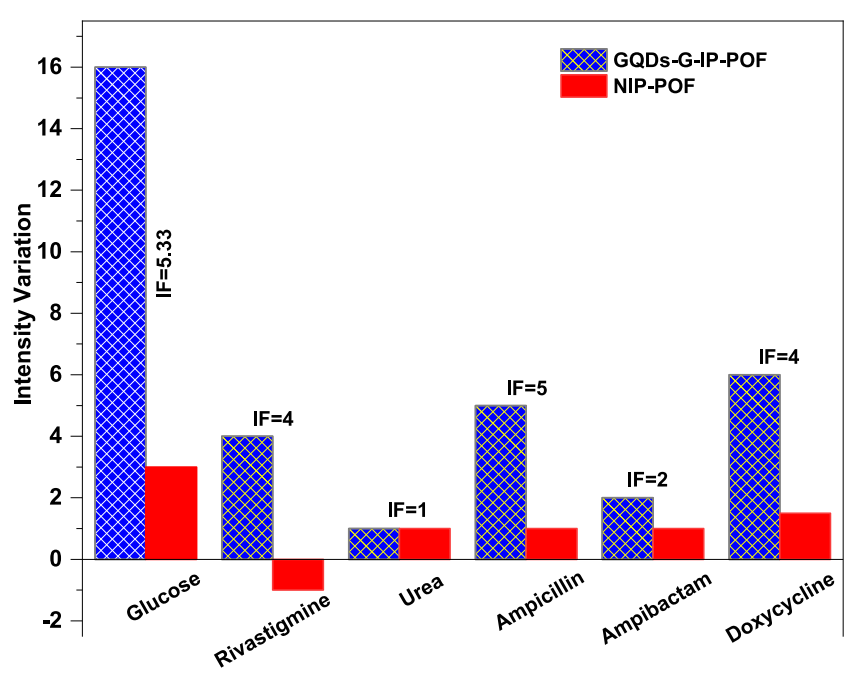

Figure 14. Selectivity of the GQDs-G-IP-coated POF toward glucose, urea, doxycycline, ampicillin, rivastigmine, and ampibactam.

ranging from 0 to $30 \mu \mathrm{M}$ were prepared and poured into the flow cell successively at room temperature. Note that every intensity was recorded after only $5 \mathrm{~s}$ of pouring the solution by a very soft tip syringe to control the volume accompanied by diminishing the pressure on the measurement, as we then represent this time as a response time of sensing probes. The performance of the as-prepared sensors with different surface roughnesses of optical fiber with a diameter of $1 \mathrm{~mm}$ for the detection of glucose solutions is shown in Figure 9. The experimental competitive studies clearly present the effect of surface roughness on the output light intensity and sensitivity response. $^{38,39}$

Consequently, the variation of transmitted light intensity of the coated POF is significantly affected by the attenuation of evanescent waves intensity manipulated by surface roughness in the sensing region. Note that the rest of our measurement was carried out with etched optical fibers with 2000 grit sandpaper. The other point of concern was the percentage of etching diameter of optical fiber; $40 \%$ was applied for all different diameters of optical fiber, as reported in our previous work, introducing the optimized power efficiency of the geometric-based features. ${ }^{32}$

After optimizing the surface roughness of the optical fiber, for the characterization of the effects of functionalized coatings accompanied by that of optical fiber diameter, various fabricated probes were fixed into the flow cell, as illustrated in the experimental setup (see Figure 1). The light intensity variation of NIP-, G-IG-, and GQDs-G-IP-coated POFs with various diameters of $0.5,0.75$, and $1 \mathrm{~mm}$ is displayed and compared in Figure $11 \mathrm{~b}-\mathrm{d}$ versus different concentrations of glucose solutions. This result implies that the output light intensity of all fibers coated with active layers increased with increasing glucose concentration. This is because, as the concentration of glucose increases in the solution, the RI difference between the coated fibers and glucose solution decreases. ${ }^{32}$ Moreover, the attenuation of the evanescent wave 
Table 1. Comparison of Enzymatic and Nonenzymatic Glucose Sensor Performance

\begin{tabular}{|c|c|c|c|c|}
\hline technique method & sensing layer & linear range $(\mathrm{mM})$ & limit of detection $(\mu \mathrm{M})$ & references \\
\hline nonenzymatic/Cu electrode & $\mathrm{Cu}$ nanowires & $0.0004-2$ & 0.049 & 42 \\
\hline $\mathrm{GO}_{x}($ enzymatic)/electrocatalysis & polyphenanthroline & $0.05-4$ & 50 & 43 \\
\hline nonenzymatic & $\mathrm{CuO}$ nanoneedle/graphene/carbon & 5.3 & 0.10 & 44 \\
\hline nonenzymatic/electrochemical & GO-MIP & $0.01-6$ & 0.02 & 45 \\
\hline enzymatic/fiber grating & $\mathrm{GO}$ & $0-8$ & & 46 \\
\hline noninvasive method of collecting transdermal glucose & glucose binding protein (GBP) & $0.004-0.02$ & 2 & 47 \\
\hline nonenzymatic evanescent wave & GQDs-G-IP & $10-100 \mathrm{nM}$ & 0.00278 & this work \\
\hline
\end{tabular}

caused by leakage, scattering, and refraction of the light at the sensing region decreased, thereby indicating lower light absorption of coating layers upon exposure to increasing glucose concentration.

Once the sensor probe was immersed in the tested glucose solution, the GQDs-G-IP layer recognized glucose due to the complementary shape and size. The experimental sensing comparison tests by NIP and G-IP (Figure $11 \mathrm{~b}-\mathrm{d}$ ) show that the number of binding active sites can be controlled by the glucose molecules in G-IP and GQDs-G-IP, while due to the lack of active sites, an appreciable and clear trend could not be observed for NIP. Compared to the NIP and G-IP functionalization, a considerable intensity variation caused by GQDs-G-IP in the local microenvironment sensing region was observed due to exploiting graphene in the G-IP, leading to the enhancement of sensing performance. This improvement is attributed to the unique properties of GQDs-G-IP such as high electron transfer rate and high optical transparency, which lead to strong light and electron transmission to the interface between the core of the fiber to the coating layer. Furthermore, with an increase in concentration, the site availability decreases, showing a plateau in the light intensity variation as a saturating behavior at the higher concentration. ${ }^{40}$

The template molecule recognition by the binding sites of GQDs-G-IP-coated POFs with three different diameters of $0.5,0.75$, and $1.0 \mathrm{~mm}$ was compared in more detail (Figure 12). In the low concentration range of $10-100 \mathrm{nM}$, the GQDs-G-IP-coated POFs with 0.75 and $1.0 \mathrm{~mm}$ diameters exhibit nearly similar linear sensing response against glucose concentration. The range of sensitivity and its value are closely related to the coverage area of the sensing region and the number of active sites of MIP. Because the fiber with a larger diameter provides more reactive support for GQDs-G-IP coating and subsequently changes interaction, sensitivity and repeatability were increased. Moreover, it renders more intense light in the sensing region, which leads to the enhanced manipulation of light variation corresponding to the binding of the glucose molecule as well as increase of $R^{2}$ and sensitivity.

The inter and intraday repeatability of the as-prepared GQDs-G-IP-POF sensor was investigated in $50 \mathrm{nM}$ glucose five times. The relative standard deviation (RSD\%) was as low as 0.0377 and $3.77 \%$ for entering day and intraday, respectively. Also, the reproducibility test was conducted for five sensors at $50 \mathrm{nM}$ glucose concentration, and the RSD \% was found to be 0.0476 for the GQDs-G-IP-POF sensor. After 60 days, the GQDs-G-IP-POF response remained 98\%, which confirmed and validated the outstanding repeatability and stability of the proposed GQDs-G-IP-POF sensor. The estimated response time is a few seconds (around $5 \mathrm{~s}$ ), as shown in Figure 13a, which is the duration of observing the light intensity variation for the two steps of background DI water and glucose solution into the flow cell. Achievement of successive measurement requires that the outlet be opened and exposed the probe to the DI water to fully wash and recovered back to its stable reference intensity. The reusability of the sensing probes was determined by successive measurements on GQDs-G-IP-coated POF with different nanomolar glucose concentrations (with nearly $10 \mathrm{~min}$ rest between each measurement). The obtained results of sensing experiments and the short response time exploiting only one sensing probe in the flow cell signify usability toward real-time low concentration detection with continuous usage. Furthermore, $94 \%$ of the initial response of the prepared GQDs-G-IPcoated POF sensor was maintained even after 9 weeks, which indicates that the proposed sensor possesses good reproducibility and stability adding up to its high sensitivity in a trace level of glucose concentration.

The high selectivity of the sensing probe is an advantage for the analyte and is also a defining characteristic of exploiting the molecular imprinting technique. To assess the as-prepared probe selectivity, experiments were also performed on the NIPand G-IP-coated POF probes for glucose detection in the presence of urea, doxycycline, ampicillin, rivastigmine, and ampibactam at $50 \mathrm{nM}$ concentration (Figure 14). Since the glucose concentration in physiological fluids is $\sim 10$ times higher than that of saccharides, namely, fructose, mannose, maltose, galactose, sucrose, lactose, and xylose, the mentioned interfering substances do not interfere with glucose detection. Thus, we proposed other applicable and size similarity compounds, which can be applied for the detection of glucose in water samples. It can be concluded that the binding sites prepared in the G-IP layer were complementary to the shape and size of only glucose molecules. The imprinting factor (IF), which expresses the ratio of the sensor response of the target under specific-to-nonspecific binding ( $I F=\mathrm{RI}_{\mathrm{MIP}} / \mathrm{RI}_{\mathrm{NIP}}$ ), was investigated as an important parameter in evaluating the effectiveness of the imprinting process (Figure 14). The high IF values demonstrate the outstanding selectivity of this prepared sensor, which can be mainly due to shape identification and electrostatic polymer structure. ${ }^{41}$

The limit of detection (LOD) and limit of quantification (LOQ) of the as-prepared sensor probes, which represent the detectable amount of near-zero analyte concentration, were calculated based on $3 \mathrm{Sb} /$ slope and $10 \mathrm{Sb}$ /slope ( $\mathrm{Sb}$ is the standard deviation of the blank samples), respectively. The LOD was found to be 2.784 and $5.3 \mathrm{nM}$ for GQDs-G-IP- and G-IP-coated POF with a diameter of $1 \mathrm{~mm}$, respectively. To clarify the improvement of the low concentration of glucose detection, the LODs of the various sensors are listed and compared in Table 1. This summarized list exhibits the least value of LOD as well as a very short response time of our proposed glucose sensor over other fiber sensors, which makes the proposed real-time sensor advantageous to others. 


\section{CONCLUSIONS}

Integrating the advantages of natural-sourced GQD nanosheets, molecular imprinting polymer, and large-core polymer optical fibers, we prepared a GQDs-G-IP-sensitive probe for glucose detection. Incorporating GQDs into the G-IP matrix manifests typical rapid response to glucose, exhibiting not only comparable performances to previously reported sensors but also high selectivity, repeatability, and ability for continuous real-time glucose detection. It is believed that the fabrication of this biosensor could promote the development of a fiber-optic glucose sensor. Since linear response range was still restricted due to the small volume of the sensing region, we will focus on the optimization of the optical fiber sensing range performance through the improvement of surface area and porous properties of the sensing MIP layer.

\section{ASSOCIATED CONTENT}

\section{SI Supporting Information}

The Supporting Information is available free of charge at https://pubs.acs.org/doi/10.1021/acsomega.0c01908.

Optical microscope image characterizations of bare optical fiber polished by 2000 grit sandpaper (Figure S1) and line graph of the $y$ component of electric field norm distribution profiles of bare and coated optical fibers (Figure S2) (PDF)

\section{AUTHOR INFORMATION}

\section{Corresponding Authors}

H. Ali Navid - Department of Laser and Optical Engineering, University of Bonab, Bonab 5551761167, Iran;

Email: navid.ali93@gmail.com

Roghaieh Parvizi - Department of Physics, College of Sciences, Yasouj University, Yasouj 75914-353, Iran; 이이이.org/00000002-2849-7771; Email: parvizi.r@yu.ac.ir

\section{Authors}

Tahereh Azargoshasb - Department of Laser and Optical Engineering, University of Bonab, Bonab 5551761167, Iran

Hadi Heidari - School of Engineering, University of Glasgow, Glasgow G12 8QQ, United Kingdom; 이이.org/00000001-8412-8164

Complete contact information is available at: https://pubs.acs.org/10.1021/acsomega.0c01908

\section{Author Contributions}

T.A. carried out the synthesizing and physical/optical sensing experiments; H.A.N. supervised, reviewed, and edited the original draft; and R.P. proposed the idea, supervised the experiments, and wrote the main manuscript text. H.H. helped in the data analysis and edited the manuscript. All authors reviewed the manuscript.

\section{Notes}

The authors declare no competing financial interest.

\section{ACKNOWLEDGMENTS}

This work was partially supported by the UK EPSRC under grant EP/R511705/1 and also the Research Council of the University of Yasouj (grant no. Gryu-89131107). The authors thank Alireza Nikzad (Yasouj University) for synthesis and Saeed Azad (École de Technologie Supérieure Montréal, Canada) for optical setups.

\section{REFERENCES}

(1) Radhakumary, C.; Sreenivasan, K. Naked eye detection of glucose in urine using glucose oxidase immobilized gold nanoparticles. Anal. Chem. 2011, 83, 2829-2833.

(2) Tang, J.; Wang, Y.; Li, J.; Da, P.; Geng, J.; Zheng, G. Sensitive enzymatic glucose detection by $\mathrm{TiO}_{2}$ nanowire photoelectrochemical biosensors. J. Mater. Chem. A 2014, 2, 6153-6157.

(3) Ayenimo, J. G.; Adeloju, S. B. Improved potentiometric glucose detection with ultra-thin polypyrrole-glucose oxidase films. Anal. Methods 2014, 6, 8996-9006.

(4) Kim, Y.-J.; Saviers, K. R.; Fisher, T. S.; Irazoqui, P. P. Continuous glucose monitoring with a flexible biosensor and wireless data acquisition system. Sens. Actuators, B 2018, 275, 237-243.

(5) Kalaivani, G. J.; Suja, S. Nanomolar level sensing of glucose in food samples using glucose oxidase confined mwcnt-inulin- $\mathrm{TiO}_{2}$ bionanocomposite. Food Chem. 2019, 298, No. 124981.

(6) Kim, N.-Y.; Adhikari, K. K.; Dhakal, R.; Chuluunbaatar, Z.; Wang, C.; Kim, E.-S. Rapid, sensitive, and reusable detection of glucose by a robust radiofrequency integrated passive device biosensor chip. Sci. Rep. 2015, 5, No. 7807.

(7) Benson, J.; Fung, C. M.; Lloyd, J. S.; Deganello, D.; Smith, N. A.; Teng, K. S. Direct patterning of gold nanoparticles using flexographic printing for biosensing applications. Nanoscale Res. Lett. 2015, 10, No. 127.

(8) Kottmann, J.; Grob, U.; Rey, J.; Sigrist, M. Mid-infrared fibercoupled photoacoustic sensor for biomedical applications. Sensors 2013, 13, 535-549.

(9) Torul, H.; Çiftçi, H.; Çetin, D.; Suludere, Z.; Boyacı, I. H.; Tamer, U. Paper membrane-based SERS platform for the determination of glucose in blood samples. Anal. Bioanal. Chem. 2015, 407, $8243-8251$.

(10) Brüggemann, O. Catalytically active polymers obtained by molecular imprinting and their application in chemical reaction engineering. Biomol. Eng. 2001, 18, 1-7.

(11) Grieshaber, D.; MacKenzie, R.; Vörös, J.; Reimhult, E. Electrochemical biosensors-sensor principles and architectures. Sensors 2008, 8, 1400-1458.

(12) Pahurkar, V. G.; Tamgadge, Y.; Gambhire, A.; Muley, G. Evanescent wave absorption based polyaniline cladding modified fiber optic intrinsic biosensor for glucose sensing application. Measurement 2015, 61, 9-15.

(13) Bosch, M.; Sánchez, A.; Rojas, F.; Ojeda, C. Recent development in optical fiber biosensors. Sensors 2007, 7, 797-859.

(14) De Luca, P.; Lepore, M.; Portaccio, M.; Esposito, R.; Rossi, S.; Bencivenga, U.; Mita, D. Glucose determination by means of steadystate and time-course UV fluorescence in free or immobilized glucose oxidase. Sensors 2007, 7, 2612-2625.

(15) Song, E.; Choi, J.-W. Conducting polyaniline nanowire and its applications in chemiresistive sensing. Nanomaterials 2013, 3, 498523.

(16) Movlaee, K.; Raza, H.; Pinna, N.; Leonardi, S.; Neri, G. Ultrasensitive Non-enzymatic Electrochemical Glucose Sensor Based on $\mathrm{NiO} / \mathrm{CNT}$ Composite. In Convegno Nazionale Sensori; Springer, 2018; pp 135-140.

(17) Gui, R.; Jin, H.; Guo, H.; Wang, Z. Recent advances and future prospects in molecularly imprinted polymers-based electrochemical biosensors. Biosens. Bioelectron. 2018, 100, 56-70.

(18) Zhao, W.; Zhang, R.; Xu, S.; Cai, J.; Zhu, X.; Zhu, Y.; Wei, W.; Liu, X.; Luo, J. Molecularly imprinted polymeric nanoparticles decorated with $\mathrm{Au}$ NPs for highly sensitive and selective glucose detection. Biosens. Bioelectron. 2018, 100, 497-503.

(19) Weber, P.; Riegger, B. R.; Niedergall, K.; Tovar, G. E.; Bach, M.; Gauglitz, G. Nano-MIP based sensor for penicillin G: Sensitive layer and analytical validation. Sens. Actuators, B 2018, 267, 26-33.

(20) Okutucu, B.; Önal, S. Molecularly imprinted polymers for separation of various sugars from human urine. Talanta 2011, 87, 7479 .

(21) Xu, Z.; Kuang, D.; Zhang, F.; Tang, S.; Deng, P.; Li, J. Fluorogenic molecularly imprinted polymers with double recognition 
abilities synthesized via click chemistry. J. Mater. Chem. B 2013, 1, $1852-1859$.

(22) Janáky, C.; Rajeshwar, K. The role of (photo) electrochemistry in the rational design of hybrid conducting polymer/semiconductor assemblies: From fundamental concepts to practical applications. Prog. Polym. Sci. 2015, 43, 96-135.

(23) Yang, X.; Gao, Y.; Ji, Z.; Zhu, L.-B.; Yang, C.; Zhao, Y.; Shu, Y.; Jin, D.; Xu, Q.; Zhao, W.-W. Dual Functional Molecular Imprinted Polymer-Modified Organometal Lead Halide Perovskite: Synthesis and Application for Photoelectrochemical Sensing of Salicylic Acid. Anal. Chem. 2019, 91, 9356-9360.

(24) Bagheri, H.; Ayazi, Z.; Naderi, M. Conductive polymer-based microextraction methods: a review. Anal. Chim. Acta 2013, 767, 113.

(25) Geim, A. K. Graphene: status and prospects. Science 2009, 324, $1530-1534$

(26) Dan, Y.; Lu, Y.; Kybert, N. J.; Luo, Z.; Johnson, A. C. Intrinsic response of graphene vapor sensors. Nano Lett. 2009, 9, 1472-1475.

(27) Neto, A. H. C.; Guinea, F.; Peres, N. M.; Novoselov, K. S.; Geim, A. K. The electronic properties of graphene. Rev. Mod. Phys. 2009, 81, No. 109.

(28) Yuan, W.; Shi, G. Graphene-based gas sensors. J. Mater. Chem. A 2013, 1, 10078-10091.

(29) Hernaez, M.; Zamarreño, C.; Melendi-Espina, S.; Bird, L.; Mayes, A.; Arregui, F. Optical fibre sensors using graphene-based materials: A review. Sensors 2017, 17, No. 155.

(30) Wu, H.; Tian, Q.; Zheng, W.; Jiang, Y.; Xu, J.; Li, X.; Zhang, W.; Qiu, F. Non-enzymatic glucose sensor based on molecularly imprinted polymer: A theoretical, strategy fabrication and application. J. Solid State Electrochem. 2019, 23, 1379-1388.

(31) Yu, S.; Ding, L.; Lin, H.; Wu, W.; Huang, J. A novel optical fiber glucose biosensor based on carbon quantum dots-glucose oxidase/cellulose acetate complex sensitive film. Biosens. Bioelectron. 2019, 146, No. 111760.

(32) Parvizi, R.; Azad, S.; Dashtian, K.; Ghaedi, M.; Heidari, H. Natural source-based graphene as sensitising agents for air quality monitoring. Sci. Rep. 2019, 9, No. 3798.

(33) Chen, P.; Liang, X.; Wang, J.; Zhang, D.; Yang, S.; Wu, W.; Zhang, W.; Fan, X.; Zhang, D. PEO/PVDF-based gel polymer electrolyte by incorporating nano- $\mathrm{TiO}_{2}$ for electrochromic glass. J. SolGel Sci. Technol. 2017, 81, 850-858.

(34) Cormack, P. A. G.; Elorza, A. Z. Molecularly imprinted polymers: synthesis and characterisation. J. Chromatogr. B 2004, 804, $173-182$.

(35) Sobral, H.; Peña-Gomar, M. Determination of the refractive index of glucose-ethanol-water mixtures using spectroscopic refractometry near the critical angle. Appl. Opt. 2015, 54, 8453-8458.

(36) Zu, P.; Chan, C. C.; Lew, W. S.; Jin, Y.; Zhang, Y.; Liew, H. F.; Chen, L. H.; Wong, W. C.; Dong, X. Magneto-optical fiber sensor based on magnetic fluid. Opt. Lett. 2012, 37, 398-400.

(37) Lee, B. H.; Kim, Y. H.; Park, K. S.; Eom, J. B.; Kim, M. J.; Rho, B. S.; Choi, H. Y. Interferometric fiber optic sensors. Sensors 2012, 12, 2467-2486.

(38) Zhong, N.; Zhu, X.; Liao, Q.; Wang, Y.; Chen, R.; Sun, Y. Effects of surface roughness on optical properties and sensitivity of fiber-optic evanescent wave sensors. Appl. Opt. 2013, 52, 3937-3945.

(39) Polynkin, P.; Polynkin, A.; Peyghambarian, N.; Mansuripur, M. Evanescent field-based optical fiber sensing device for measuring the refractive index of liquids in microfluidic channels. Opt. Lett. 2005, 30, 1273-1275.

(40) Shrivastav, A. M.; Usha, S. P.; Gupta, B. D. Fiber optic profenofos sensor based on surface plasmon resonance technique and molecular imprinting. Biosens. Bioelectron. 2016, 79, 150-157.

(41) Vasapollo, G.; Sole, R. D.; Mergola, L.; Lazzoi, M. R.; Scardino, A.; Scorrano, S.; Mele, G. Molecularly imprinted polymers: present and future prospective. Int. J. Mol. Sci. 2011, 12, 5908-5945.

(42) Zhuang, Z.; Su, X.; Yuan, H.; Sun, Q.; Xiao, D.; Choi, M. M. An improved sensitivity non-enzymatic glucose sensor based on a $\mathrm{CuO}$ nanowire modified $\mathrm{Cu}$ electrode. Analyst 2008, 133, 126-132.
(43) Oztekin, Y.; Ramanaviciene, A.; Yazicigil, Z.; Solak, A. O.; Ramanavicius, A. Direct electron transfer from glucose oxidase immobilized on polyphenanthroline-modified glassy carbon electrode. Biosens. Bioelectron. 2011, 26, 2541-2546.

(44) Ye, D.; Liang, G.; Li, H.; Luo, J.; Zhang, S.; Chen, H.; Kong, J. A novel nonenzymatic sensor based on $\mathrm{CuO}$ nanoneedle/graphene/ carbon nanofiber modified electrode for probing glucose in saliva. Talanta 2013, 116, 223-230.

(45) Alexander, S.; Baraneedharan, P.; Balasubrahmanyan, S.; Ramaprabhu, S. Highly sensitive and selective non enzymatic electrochemical glucose sensors based on Graphene Oxide-Molecular Imprinted Polymer. Mater. Sci. Eng., C 2017, 78, 124-129.

(46) Jiang, B.; Zhou, K.; Wang, C.; Sun, Q.; Yin, G.; Tai, Z.; Wilson, K.; Zhao, J.; Zhang, L. Label-free glucose biosensor based on enzymatic graphene oxide-functionalized tilted fiber grating. Sens. Actuators, B 2018, 254, 1033-1039.

(47) Tiangco, C.; Fon, D.; Sardesai, N.; Kostov, Y.; Sevilla, F., III; Rao, G.; Tolosa, L. Fiber optic biosensor for transdermal glucose based on the glucose binding protein. Sens. Actuators, B 2017, 242, $569-576$. 